\title{
MAPAS CONCEITUAIS COMO FERRAMENTA FACILITADORA DA APRENDIZAGEM DO ENSINO DE QUÍMICA ORGÂNICA
}

\author{
Suiane Costa Alves ${ }^{1}$, Esilene Reis $^{1}$, \\ Dafne Alexandre CaValcante ${ }^{1}$, Maria Goretti De VASCOncelos Silva ${ }^{1,2}$ \\ Universidade Federal do Ceará (UFC) \\ ${ }^{1}$ Mestrado Profissional em Ensino de Ciências e Matemática \\ ${ }^{2}$ Programa de Pós-Graduação em Química \\ <suianealves@yahoo.com.br>,<esilene@hotmail.com> \\ <dafne@ufc.br>.<mgvsilva@ufc.br>
}

DOI: 10.21439/conexoes.v9i4.963

\begin{abstract}
Resumo. O presente trabalho relata a investigação realizada em uma turma do Ensino Médio de uma escola pública na cidade de Maracanaú - CE. Foram utilizados mapas conceituais (MCs) como ferramenta facilitadora da aprendizagem do ensino de Química. Este estudo foi norteado pela teoria da aprendizagem significativa desenvolvida por David Ausubel, produzindo resultados satisfatórios com o uso dos MCs como ferramenta na construção da aprendizagem do conteúdo abordado. Este estudo confirma que a relação entre conceitos apresentados através de MCs pode ser assimilada pelos estudantes, promovendo a aprendizagem significativa.
\end{abstract}

Palavras-chaves: Mapa conceitual. Química orgânica. Ensino de Química.

Abstract. This paper reports an investigation that performed in a high school class of a public school in Maracanaú - CE and which consists in the use of concept maps (CMs) as facilitator tool of Chemistry teaching. The theory of meaningful learning developed by David Ausubel guided this study, which produced satisfactory results with the use of CMs as a tool in the construction of the used content approach learning. This study confirms that the relationship between concepts required by CMs can be assimilated by the students, promoting meaningful learning.

Keywords: Concept maps. Organic Chemistry. Chemistry teaching.

\section{INTRODUÇÃO}

Os educadores buscam cada vez mais a aplicação de metodologias que tornem mais produtivos os processos de ensino e aprendizagem. A tendência de se aplicar metodologias de ensino que seguem a linha construtivista se evidencia nas crescentes pesquisas para o desenvolvimento de instrumentos, técnicas, metodologias ou processos no ensino, tanto em sala de aula, quanto fora dela, como no caso das aulas experimentais (ARAUJO et al., 2006). Dentre as várias estratégias em questão, os mapas conceituais (MCs) vêm se destacando e têm sido utilizados nas mais diferentes áreas do conhecimento (FREITAS FILHO, 2007).

A utilização de diferentes abordagens contribui para o desenvolvimento dos subsunçores dos alunos por meio de atividades através das quais eles possam utili- zar conceitos previamente adquiridos para a compreensão de novos assuntos. De acordo com Moreira (2013), a aprendizagem significativa compreende a incorporação de novos conhecimentos ou saberes, conferindo ao indivíduo a capacidade de refletir diante de novas situações. Essas incorporações relacionam-se com o conhecimento prévio que o aluno possui (subsunçor). Assim, uma das condições básicas para que ocorra a aprendizagem significativa é a predisposição ao aprendizado, bem como a relação deste processo com o conhecimento prévio que o educando possui. Moreira e Masini (2009) destacam que a assimilação de conhecimentos trata-se de um processo potencialmente significativo. Neste processo, o conceito ou ideia são assimilados pela estrutura cognitiva, promovendo a representação de extensão, qualificação ou complementação aos mesmos.

Genericamente, mapas conceituais (ou mapas de 
conceitos) são diagramas indicando relações entre conceitos ou entre palavras, que usamos para representar definições. São diagramas de significados, de relações significativas, de hierarquias conceituais. Esta técnica foi desenvolvida pelo pesquisador americano Joseph Novak e colaboradores na Universidade de Cornell, nos Estados Unidos, e não implica necessariamente sequência, temporalidade ou direcionalidade. Consiste em uma técnica versátil que é utilizada em várias áreas do conhecimento e para diferentes finalidades (MOREIRA, 2012).

O uso de mapas conceituais destaca o conhecimento prévio como base para novos conhecimentos, modificando o que Piaget definiu como esquemas de assimilação e acomodação do novo conhecimento. Segundo Moreira (2013, p. 17), "a visão de Novak é que a aprendizagem significativa subjaz à integração positivista construtivista de pensamentos, sentimentos e ações que levam ao engrandecimento humano".

Atividades didáticas, principalmente MCs, devido à sua grande adaptabilidade - representando uma aula, um programa educacional ou ainda a análise de artigos, capítulos de livros, experimentos ou avaliação da aprendizagem - levarão à ancoragem de conhecimentos. $\mathrm{O}$ estudante vivencia esta experiência fazendo a assimilação dos novos conceitos (TOIGO, 2012).

A aprendizagem significativa envolve a outorga de significados e, aplicada com o uso de mapas, independente de quem os tenha elaborado, são dotados de componentes pessoais. Isso significa que não existe um único nem um correto MC para representar uma situação, um conteúdo ou um experimento. O MC criado pelo estudante é importante porque ele indica se o estudante está aprendendo significativamente o conteúdo. São utilizados desde os anos setenta e, hoje atingem as várias áreas do conhecimento, atribuindo novos significados aos conceitos de ensino, aprendizagem e avaliação, sendo inseridos frequentemente nas salas de aula (TOIGO, 2012).

As diversas pesquisas acadêmicas na área de ensino de Química, com diferentes enfoques, influenciam as técnicas de ensino e de aprendizagem. Quando são incorporadas ou norteiam guias didáticos ou instruções de laboratório, por exemplo, ajudam a melhorar a compreensão dos conceitos por parte dos alunos. Técnicas gráficas como a dos mapas conceituais estão baseadas em uma perspectiva construtivista e possuem contribuições efetivas para a aprendizagem significativa; podem favorecer a reconstrução e a troca de novos significados e trazer uma maior compreensão, além de servir como recurso para avaliar o progresso do estudante (ARAUJO et al., 2006).
O desafio de alcançar um aprendizado mais significativo no Ensino de Química, usualmente considerado difícil pelos estudantes, vai de encontro à realidade da ausência de ferramentas e estratégias que tornem o ensino mais motivador. Técnicas gráficas como MCs podem ser instrumentos úteis, pois estão baseadas em uma perspectiva construtivista, além de possuírem contribuições efetivas para a aprendizagem significativa (ARAUJO et al., 2006; TRINDADE e HARTWIG, 2012). A necessidade de despertar interesse nos estudantes e de fazer uso de metodologias de ensino que promovam aprendizagem significativa, diminuindo a abstração que frequentemente povoa o ensino de Química, justifica a utilização de MCs em aulas de Química para o Ensino Médio, motivando a realização deste estudo.

\section{REVISÃO DE LITERATURA}

A utilização de mapas conceituais (MCs) é baseada principalmente na Teoria de Aprendizagem ou Teoria da Assimilação, de David Ausubel (1968). Essa teoria explica como o estudante processa e armazena o conhecimento a partir da organização hierárquica dos conceitos e suas relações, do mais geral ao mais específico. Ausubel (1982) relata que o indivíduo constrói significados a partir das relações entre um novo conceito, seu conhecimento prévio e sua predisposição para realizar essa construção (FREITAS FILHO, 2007; PELIZZARI et al., 2002).

Para Novak (2000, p.8)

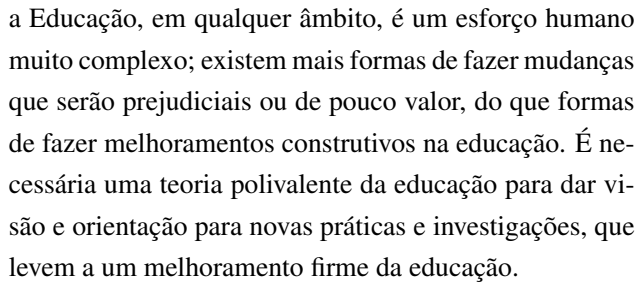

A partir do desenvolvimento dessas ideias, a aprendizagem parece ocorrer a partir de uma dinâmica de interação entre diferentes conhecimentos, culminando com a formação de uma rede cognitiva cuja estrutura é constantemente reorganizada durante a aprendizagem significativa e se mantém na memória.

Ausubel (2000) defende o seu ponto de vista sobre a temática, explicando que, o conhecimento é significativo por definição. É o pro-
duto significativo de um processo psicológico cognitivo
("saber") que envolve a interação entre ideias "logica-
mente" (culturalmente) significativas, ideias anteriores
("ancoradas") relevantes da estrutura cognitiva particular 
do aprendiz (ou estrutura dos conhecimentos deste) e o "mecanismo" mental do mesmo para aprender de forma significativa ou para adquirir e reter conhecimentos.

Já Moreira (1997, p.7) admite que a aprendizagem é significativa

quando uma nova proposição adquire significados para o aprendiz através de uma espécie de ancoragem em aspectos relevantes da estrutura cognitiva preexistente do indivíduo, isto é, em conceitos, idéias, proposições já existentes em sua estrutura de conhecimentos (ou de significados) com determinado grau de clareza, estabilidade e diferenciação.

De acordo com Tavares (2004, p.55), nos processos que envolvem a aprendizagem significativa,

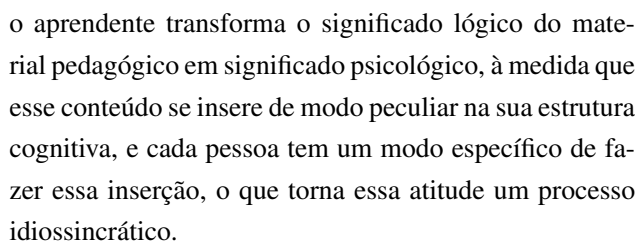

Para Ausubel et al. (1980, p. 39) existem três tipos de aprendizagem significativa: a aprendizagem representacional (que está relacionada ao aspecto de o indivíduo ser capaz de conectar o objeto ao símbolo que o representa), a aprendizagem conceitual (que ocorre quando o aluno compreende o conceito propriamente dito associado ao objeto) e a aprendizagem proposicional (trata-se daquela que se refere aos significados exprimidos por grupos de palavras combinadas em proposições ou sentenças).

Maffra (2010, p. 8) corrobora dizendo que:

a teoria de Ausubel, aponta diretrizes e princípios que levam a uma estratégia facilitadora da aprendizagem significativa e, Novak, baseado na diferenciação progressiva, desenvolve os mapas conceituais como recurso para a prática pedagógica capaz de propiciar tal forma de aprendizagem.

$\mathrm{Na}$ aprendizagem significativa, primeiramente um conhecimento novo passa a ter significado para o educando, entrando em cena o componente idiossincrático da significação. Esse processo envolve sempre a atribuição de significados e a incorporação da natureza pessoal do estudante. Sem a atribuição de significados pessoais ou a relação com um conhecimento preexistente, a aprendizagem se torna mecânica, não significativa.

Dessa maneira, observamos que os elementos organizadores admitem essencial importância na teoria da aprendizagem significativa, e Ausubel (2000, p.12) ensina que, fundamentalmente, a base lógica para o seu uso parte dos seguintes princípios:

1. A importância de se possuírem ideias relevantes, ou apropriadas, estabelecidas, já disponíveis na estrutura cognitiva, para fazer com que as novas ideias logicamente significativas se tornem potencialmente significativas e as novas ideias potencialmente significativas se tornarem realmente significativas (i.e., possuírem novos significados), bem como fornecer-lhes uma ancoragem estável.

2. As vantagens de se utilizarem as ideias mais gerais e inclusivas de uma disciplina na estrutura cognitiva como ideias ancoradas ou subsunçores, alteradas de forma adequada para uma maior particularidade de relevância para o material de instrução. Devido maior aptidão e especificidade da relevância das mesmas, também usufruem de uma maior estabilidade, poder de explicação e capacidade integradora inerentes.

3. O fato de os próprios organizadores tentarem identificar um conteúdo relevante já existente na estrutura cognitiva (e estarem explicitamente relacionados com esta) e indicar, de modo explícito, a relevância quer do conteúdo existente, quer deles próprios para o novo material de aprendizagem.

O estabelecimento de relações entre ideias, conceitos e proposições já conhecidos na estrutura cognitiva é um processo que ocorre na aprendizagem significativa, sendo denominado de relações entre subsunçores. Elementos existentes na estrutura cognitiva são percebidos e relacionados, adquirindo novos significados e conduzindo a uma reorganização da estrutura cognitiva (TOIGO, 2012).

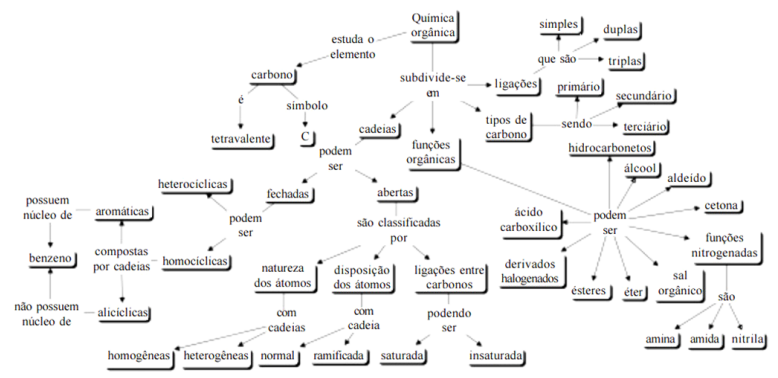

Figura 1: Exemplo de Mapa Conceitual produzido por alunos de Ensino Médio Fonte: Correia et al. (2008).

Novak (2002) desenvolveu a metodologia de MCs baseado na teoria da aprendizagem significativa, buscando representar como o conhecimento é armazenado na estrutura cognitiva. Assim, o uso de MCs exterioriza o conhecimento através de palavras de ligação, formando proposições que mostram as relações existentes entre conceitos percebidos pelo educando (FREITAS FILHO, 2007). 


\section{METODOLOGIA}

Este trabalho foi desenvolvido no horário vespertino com uma turma de 30 alunos do $3^{\circ}$ ano do Ensino Médio da Escola de Ensino Fundamental e Médio José de Borba Vasconcelos, localizada no município do Maracanaú - CE. A turma é composta por jovens que sempre estudaram em escola pública, alguns com distorção idade-série (entre 16 a 36 anos) e dois alunos portadores de necessidades especiais.

A pesquisa foi realizada em quatro etapas. A primeira foi executada em três aulas expositivas de Química, para todos os alunos, sobre funções orgânicas nitrogenadas. Ao final das aulas, os alunos foram informados sobre a pesquisa e unanimemente aceitaram participar da mesma. A segunda etapa desenvolveu-se em sala, no terceiro bimestre do ano letivo, e constou de uma revisão do conteúdo estudado na primeira etapa, e da aplicação de uma $1^{\mathrm{a}}$ avaliação (pré-teste) com cinco questões objetivas e 01 subjetiva. O pré-teste foi utilizado como forma de avaliar o conhecimento dos alunos antes da elaboração dos mapas conceituais. Todos os alunos fizeram um pré-teste que foi utilizado como base para referenciar os dados obtidos no pós-teste.

Sequencialmente, na terceira etapa, a turma foi dividida aleatoriamente em dois grupos (A e B). O grupo $\mathrm{B}$ foi apresentado à ferramenta dos $\mathrm{MCs}$ e exercitou a construção dos mapas com diferentes temáticas escolhidas pelos próprios alunos. A atividade final foi a elaboração de um MC sobre funções orgânicas nitrogenadas, tema central desta pesquisa. O grupo A não participou da oficina de elaboração de MCs. Nessa etapa, os alunos tiveram a oportunidade de, através da utilização desse recurso didático, ancorar os novos conhecimentos por meio da utilização de seus conhecimentos prévios.

Os mapas conceituais elaborados pelos alunos (do grupo B) exibiram, em sua maioria, estruturas bidimensionais nas quais se observa a apresentação do conceito geral acima e aqueles mais específicos organizados em sequência hierarquicamente inferior. Moreira (2013), dialogando sobre a aprendizagem significativa, afirma que mapas conceituais podem ser usados como recurso instrumental a fim de facilitar a compreensão de um determinado tópico disciplinar, podendo também ser usados na avaliação da aprendizagem. O momento da análise dos MCs produzidos pelos estudantes foi de extrema importância para evidenciar como os estudantes construíram e replicaram esses conhecimentos, e como reagiram diante da proposta de produzir informações através da construção de um modelo didático até então novo para eles.

Na quarta etapa da pesquisa, os alunos dos dois grupos, A e B, foram convidados a realizarem uma segunda avaliação com questões objetivas e subjetivas sobre a temática "funções nitrogenadas". O objetivo dessa avaliação foi comparar os resultados dos dois grupos, visto que somente o grupo B havia elaborado mapas conceituais com a temática em questão. Os MCs foram analisados à luz de um mapa conceitual elaborado pelos autores deste trabalho e o atendimento a quesitos previamente selecionados por sua importância (definição de funções orgânicas nitrogenadas, classificação, nomenclatura, propriedades físicas e químicas, exemplos e uso) também foi utilizado, para avaliar o aprendizado dos alunos do grupo B.

\section{APRESENTAÇÃO E DISCUSSÃO DOS DA- DOS}

Duas avaliações foram realizadas com todos os estudantes e, na $1^{\mathrm{a}}$, apenas 06 apresentaram nota superior a 50 (numa escala de 0-100), o que equivale a 20,0\% da turma. Através do resultado em questão, observa-se que apenas a aula expositiva não foi capaz de despertar o interesse da maioria da turma pelo conteúdo funções orgânicas nitrogenadas.

De acordo com a definição de aprendizagem significativa, ou seja, a incorporação de novos conhecimentos a partir do conhecimento prévio do aluno (subsunçores), justifica o uso de mapas conceituais na promoção da aprendizagem significativa. $\mathrm{Na} 2^{a}$ avaliação, 17 alunos apresentaram nota igual ou superior a 50 , sendo que desse total de alunos, 11 pertencem ao grupo B, ou seja, utilizaram MCs. Sendo assim, o rendimento dos alunos, calculado com base nos alunos em cada grupo - A (16 alunos) e B (14 alunos) - foi de $37,5 \%$ para o Grupo A e 78,6\% para o Grupo B, com relação a esse conteúdo de Química, com nota igual ou superior a 50, que é a média necessária para aprovação.

A utilização de uma metodologia didática diferenciada, em destaque, para os alunos que participaram do estudo, contribuiu para a ancoragem dos novos conhecimentos de Química, especificamente sobre o assunto abordado a respeito que dos compostos orgânicos. Diante do resultado exposto, observa-se que a aplicação do instrumental "Mapas Conceituais" potencializou o ensino, pois os alunos tiveram a oportunidade de internalizar o conhecimento, expressando-o através da confecção dos mapas.

Constatou-se que muitos alunos tiveram a sua compreensão melhorada, pois comparando as respostas obtidas no teste, verificou-se que eles apresentaram mais exemplos de compostos nitrogenados, inclusive diferenciando as amidas das aminas e suas respectivas nomenclaturas. Isso configura uma elaboração de conceitos mais completa e diferenciada, característica da dife- 
renciação progressiva, processo que ocorre no curso da aprendizagem significativa e que contribui para aquisição de novos conhecimentos e também para a atribuição de significados (MOREIRA, 1998). Abaixo, a Figura2 apresenta as notas $(0-100)$ das $1^{a}$ (a) e $2^{a}$ avaliações (b) dos Grupos A e B.

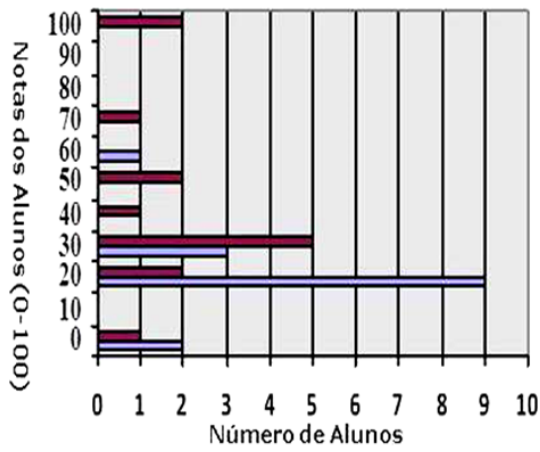

QGrupo.t QGrupo B

(a)

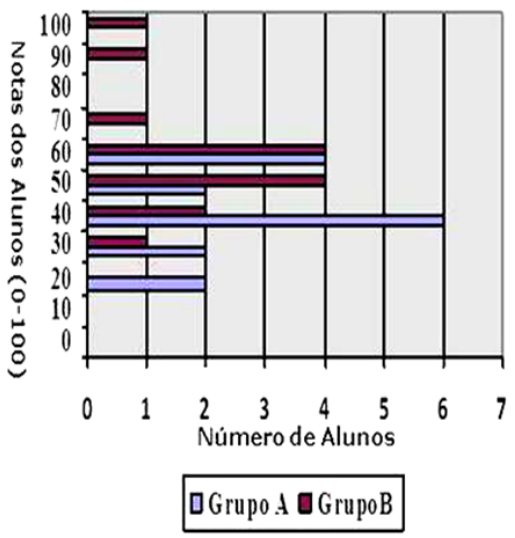

(b)

Figura 2: Notas das Avaliações dos Grupos A e B; a) notas obtidas pelos estudantes $\mathrm{x}$ número de alunos, na $1^{\mathrm{a}}$ avaliação e b) notas obtidas pelos estudantes x número de alunos, na $2^{\mathrm{a}}$ avaliação.

A partir da análise dos dados obtidos, observou-se que o uso de mapas conceituais aplicados ao conteúdo "Compostos Orgânicos Nitrogenados" promoveu resultado satisfatório, uma vez que as notas dos alunos na segunda avaliação apresentaram uma melhora significativa. Na primeira avaliação, o número de alunos que obteve nota igual ou inferior a 30 representava mais de $57 \%$ do Grupo B e depois da participação nas aulas com o uso dos MCs, o número de alunos com esta porcentagem de acerto na $2^{\text {a }}$ avaliação foi drasticamente reduzido, pois não houve alunos com nota igual ou inferior a dois (apenas um aluno obteve nota 30, porém, no teste anterior, esse aluno havia tirado zero)

Como se observa na figura 2(a) a aplicação da ferramenta MC foi decisiva no aumento percentual das notas entre $60-80$, tendo um aumento de $7,14 \%$ para $35,71 \%$. Atribuiu-se esse crescimento no percentual de acertos, ao fato dos mapas conceituais possibilitarem uma melhor estruturação e assimilação das informações que os educandos receberam. Novak (apud CORREA, DONNA e MALACHIAS, 2008) considera que os mapas conceituais não se resumem a um diagrama de fluxos; antes, são muitos mais do que isso: eles explicitam as relações significativas entre conceitos e que podem ser ordenados de forma hierárquica.

Um fato interessante observado nos mapas dos alunos é que alguns utilizaram outros exemplos de compostos orgânicos nitrogenados encontrados na natureza, diferentes daqueles citados no livro didático, como é o caso de um estudante, que em seu MC mencionou a presença da ureia no adubo e sua utilização na alimentação do gado (Figura 3). Neste caso, podemos afirmar que o aluno relacionou o conhecimento adquirido com o conhecimento que ele já possuía. Autores como Novak e Moreira (apud FREITAS FILHO, 2007) recomendam aos professores a utilização de mapas conceituais com recurso didático para identificar significados (subsunçores) pré-existentes na estrutura cognitiva do estudante e que são necessários à aprendizagem. Sabemos que nas provas tradicionais, a escrita dos alunos está condicionada aos comandos das questões, ou seja, ele registra apenas o que lhe foi solicitado, o que não ocorre nos MCs.

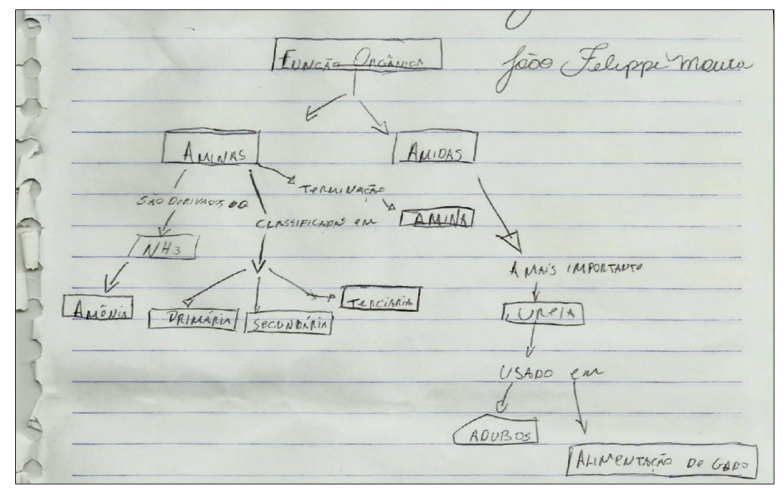

Figura 3: Mapa conceitual construído por um aluno do $3^{\circ}$ ano do Ensino Médio da Escola de Ensino Fundamental e Médio José de Borba Vasconcelos, Maracanaú - CE. Fonte: Os autores, 2015.

Os MCs podem apresentar formatos uni, bi e tridimensionais. Os estudantes utilizaram as formas uni (25\%) e bidimensional (75\%). Freitas Filho (2007, p.87) afirma: "Na prática, porém, por serem mais ela- 
borados que os unidimensionais e mais simples que os tridimensionais, os mapas bidimensionais são os mais usados".

Observou-se que apenas um estudante citou características físico-químicas das funções orgânicas nitrogenadas, resultado que pode ser considerado como um indicativo de que podem não ter compreendido esta parte do conteúdo. Este fato pode inclusive ser repensado pelo professor para que, em sala de aula, tire as dúvidas dos estudantes nesse quesito. Moreira (1998) ressalta a importância de ensinar usando organizadores prévios para fazer as pontes entre os significados que o aluno já tem e os que ele precisa ter para aprender significativamente os conteúdos.

Com relação à avaliação da aprendizagem através dos mapas conceituais (MCs), Correia, Donna e Malachias (2008, p. 485) afirmam que "a avaliação por meio de MCs não ocorre com a intenção de testar conhecimentos e atribuir nota aos alunos para classificálos, mas sim com o objetivo de obter informações sobre o tipo de estrutura que o aluno estabelece para um dado conjunto de conceitos".

\section{CONSIDERAÇÕES FINAIS}

Os Parâmetros Curriculares Nacionais (PCNs) e as Diretrizes Curriculares Nacionais para o Ensino Médio (DCNEM) têm por finalidade o desenvolvimento da autonomia intelectual e do pensamento crítico do estudante, estimulando a sensibilidade, o espírito inventivo e a capacidade de refletir e mudar a realidade a partir de reflexões sobre as problemáticas que se apresentam na sociedade contemporânea. De tal forma, o aprendizado do conteúdo de Química passa a ser um eixo integrador, quando trabalhado conjuntamente com as demais áreas do conhecimento. A partir daí é possível explicar, compreender e intervir no processo de construção do conhecimento, desenvolvendo competências e habilidades, processo que pode ser compreendido também como a relação entre pensamento reflexivo e a linguagem, propiciando as relações sociais que acabam por promover o desenvolvimento da aprendizagem.

As reflexões em torno de como promover uma aprendizagem significativa vêm sendo aprofundadas através do uso de diferentes metodologias de aprendizagem que permitam desenvolver competências e habilidades nos alunos.

Este estudo demonstrou a importância da utilização de mapas conceituais como recurso didático em aulas de Química. Observou-se uma evolução dos alunos que tiveram contato com esta metodologia, o que permitiu um aprendizado maior por parte daqueles que participaram do minicurso ministrado.
Mediante a aplicação dos MCs para o ensino das funções orgânicas nitrogenadas, percebe-se que os alunos que tiveram a oportunidade de participar da construção dos mesmos obtiveram nota em sua avaliação superior à dos alunos que não participaram da aplicação dessa metodologia. Daí a reflexão dos educadores em buscar metodologias diferenciadas que permitam aos educandos explorar seus potenciais cognitivos em busca de uma aprendizagem prazerosa que permita reflexões sobre as problemáticas que se apresentam.

$\mathrm{O}$ aumento na motivação dos alunos diante de novas estratégias de ensino também contribui para que estejam dispostos ao aprendizado. Diante dessa realidade, delineiam-se os desafios da escola sobre esse tema na tentativa de desenvolver habilidades que permitam uma maior interação entre os estudantes e educadores, bem como garantir a transposição do conhecimento, permitindo o diálogo entre as disciplinas.

\section{REFERÊNCIAS}

ARAÚJO, N. R. S. de; BUENO, E. A. S.; ALMEIDA, F. A. de S.; BORSATO, D. O petróleo e sua destilação: uma abordagem experimental no ensino médio utilizando mapas conceituais. Semina: Ciências Exatas e Tecnológicas, v. 27, n. 1, p. 57-62, 2006.

AUSUBEL, D. Educational Psychology: A Cognitive View. New York and Toronto: Holt, Rinehart and Winston, 1968.

A aprendizagem significativa: a teoria de David Ausubel. São Paulo: Moraes, 1982.

Aquisição e retenção de conhecimentos: uma perspectiva cognitiva. Lisboa: Editora Plátano, 2000.

\author{
AUSUBEL, D.; NOVAK, J. D.; HANESIAN, \\ H. Psicologia educacional. Rio de Janeiro: \\ Interamericana, 1980. Tradução Eva Nick.
}

CORREIA, P. R.; JR, J. W. D.; INFANTEMALACHIAS, M. E. Mapeamento conceitual como estratégia para romper fronteiras disciplinares: A isomeria nos sistemas biológicos concept mapping as a tool to break disciplinary boundaries: isomerism in biological systems. Ciência \& Educação, SciELO Brasil, v. 14, n. 3, p. 483-95, 2008.

FREITAS FILHO, J. R. de. Mapas conceituais: estratégia pedagógica para construção de conceitos na disciplina química orgânica. Ciências \& Cognição, v. 12, p. 86-95, 2007. 
MAFFRA, S. O uso dos mapas conceituais como recurso didático pedagógico facilitador do processo de ensino aprendizagem. Monografia (Trabalho de Conclusão de Pós-Graduação Lato Sensu) - Centro Federal de Educação Tecnológica Celso Suckow da Fonseca, 2010.

MOREIRA, M. A. A pesquisa em educação em ciências e a formação permanente do professor de ciências. In: SANCHEZ, J. M.; ONORBE, T. Y.; BUSTAMANTE, G. L. (Ed.). Educación Científica. Espanha: Servicio de Publicaciones de la Universidad de Alcalá, 1998.

Mapas conceituais e aprendizagem significativa. 2012. Disponível em: <http: //www.if.ufrgs.br/ moreira/mapasport.pdf $>$. Acesso em: 22 mar. 2016.

Aprendizagem significativa em mapas conceituais. Porto Alegre: Ed. UFRGS, Instituto de Física, 2013.

MOREIRA, M. A.; MASINI, E. F. S. Aprendizagem significativa: a teoria de aprendizagem de David Ausubel. 2. ed. São Paulo: Centauro Editora, 2009.

NOVAK, J. D. Apreender, Criar e Utilizar o Conhecimento. Mapas Conceptuais como Ferramentas de Facilitação nas Escolas e Empresas. Lisboa: Editora Plátano, 2000.

Meaningful learning: The essential factor for conceptual change in limited or inappropriate propositional hierarchies leading to empowerment of learners. Science education, Wiley Online Library, v. 86, n. 4, p. 548-571, 2002.

PELIZZARI, A.; KRIEGL, M. d. L.; BARON, M. P.; FINCK, N. T. L.; DOROCINSKI, S. I. Teoria da aprendizagem significativa segundo ausubel. revista $P E C$, v. 2, n. 1, p. 37-42, 2002.

TAVARES, R. Aprendizagem significativa. Revista conceitos, v. 55, n. 10, 2004. Disponível em: <http: //www.fisica.ufpb.br/ romero/objetosaprendizagem/ Rived/Artigos/2004-RevistaConceitos.pdf> Acesso em: 13 dez. 2015.

TOIGO, A. M.; MOREIRA, M. A.; COSTA, S. S. C. da. Revisión de la literatura sobre el uso de mapas conceptuales como estrategia didáctica y de evaluación (a review about the use of concept maps as learning and evaluation strategy). Investigações em Ensino de Ciências, v. 17, n. 2, p. 305-339, 2012. 